FERNANDES, T.F. e BRAGA, G.M.S. Obtenção de extratos liquênicos do Cerrado Maranhense para avaliação da atividade carrapaticida. PUBVET, Londrina, V. 8, N. 21, Ed. 270, Art. 1799, Novembro, 2014.

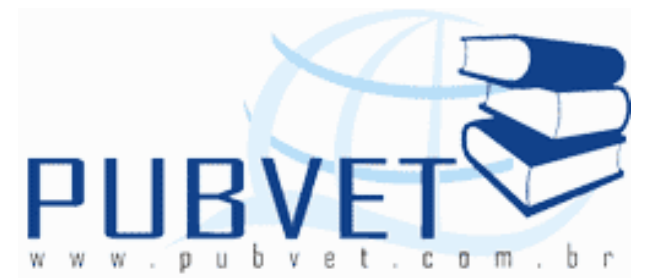

PUBVET, Publicações em Medicina Veterinária e Zootecnia.

\title{
Obtenção de extratos liquênicos do Cerrado Maranhense para avaliação da atividade carrapaticida
}

Thayane Ferreira Fernandes ${ }^{1}$, Geovania Maria da Silva Braga ${ }^{2}$.

1 Pós Graduada em Saúde Pública, CESI, Universidade Estadual do Maranhão E-mail: thayanephn@hotmail.com

2 Profa Adjunta IV, CESI/CESP, Universidade Estadual do Maranhão - UEMA, Imperatriz, Maranhão, Brasil.

\section{Resumo}

O uso de produtos químicos, na ação contra ectoparasitas vem se avolumando no Brasil. A dependência dos mesmos traz consigo, vários impactos ambientais e uma necessidade de maior concentração destes produtos, que podem ser os inseticidas, os acaricidas, ou os carrapaticidas. Dentro dos métodos alternativos de planejamento do controle de artrópodes, os liquens é uma delas. Com o objetivo de avaliar a atividade dos mesmos, houve a extração de princípios ativos dos liquens coletados, nas adjacências do município de Imperatriz, Maranhão. Através de reagentes orgânicos obtiveram-se os extratos liquênicos para posterior utilização em artrópodes. Foram realizados três testes nas espécies de carrapatos Rhipicephalus sanguineus, Rhipicephalus (Boophilus) microplus e Anocentor sp. onde foram submetidas a banhos de imersão em solução de extratos liquênicos e em solução controle. De acordo com os testes realizados foi possível observar que, os extratos liquênicos 
FERNANDES, T.F. e BRAGA, G.M.S. Obtenção de extratos liquênicos do Cerrado Maranhense para avaliação da atividade carrapaticida. PUBVET, Londrina, V. 8, N. 21, Ed. 270, Art. 1799, Novembro, 2014.

pesquisados tiveram eficácia quando aplicados nas citadas espécies de carrapatos.

Palavras-chave: Liquens. Controle. Eficácia, Carrapatos.

\title{
Obtaining from Extracts of Lichens the Cerrado Maranhense for evaluation of Activity Tickicide
}

\begin{abstract}
The use of chemicals, in action against ectoparasites comes swelled in Brazil. The dependence of these brings various environmental impacts and a need for greater concentration of these products that can result in death to insect, mites or ticks. Within the planning alternative methods of control of arthropods, lichens are one of them. In order to evaluate the activity of the same, there was the extraction of active principles of lichens collected in the vicinity of the city of Imperatriz, Maranhão. Using organic reagents lichens extracts obtained for later use in arthropods. Three tests were conducted on the species of ticks Rhipicephalus sanguineus, Rhipicephalus (Boophilus) microplus and Anocentor sp. where they were subjected to immersion baths in lichens extracts solution and in control solution. According to the tests it was possible to observe that the liquênicos extracts surveyed had efficacy when used in cited species of ticks.
\end{abstract}

Keywords: Lichens. Control. Effectiveness. Ticks.

\section{INTRODUÇÃO}

A dependência do controle químico vinculado aos problemas de impacto ambiental, custos e surtos de resistência, enfatiza as necessidades de pesquisa e alternativas viáveis do controle a determinados organismos.

Entre estes, se encontra os ectoparasitas, como os carrapatos, aracnídeos dos mais importantes nas perdas produtivas e econômicas, tanto para a pecuária em nível nacional, independente do sistema de produção, 
FERNANDES, T.F. e BRAGA, G.M.S. Obtenção de extratos liquênicos do Cerrado Maranhense para avaliação da atividade carrapaticida. PUBVET, Londrina, V. 8, N. 21, Ed. 270, Art. 1799, Novembro, 2014.

como também para os animais domésticos, que convivem com o homem, causando problemas praticamente a todos.

Para se evitar o avanço deste tipo de parasitismo, existe a utilização de várias drogas carrapaticidas, que abrange a maneira clássica e habitual de controlar as implicações que os mesmos venham a causar no crescimento dos bovinos (SOUTELLO et al., 2002).

Existem, aproximadamente, 870 espécies de carrapatos descritas no mundo, todas agrupadas na Subordem Ixodidae. A fauna brasileira de Ixodidae está atualmente representada por 61 espécies de carrapatos, com algumas classes importantes para a Saúde Pública e Veterinária, por causarem danos diretos durante sua alimentação e por poderem transmitir agentes infecciosos, aos homens e animais (BARROS-BATTESTI et al., 2006).

A região Nordeste é uma área de difícil controle de carrapatos, devido à extensa modificação climática, notadamente com reverência as precipitações atmosféricas e umidade relativa resultante e da diversidade do uso da terra para a agricultura e pecuária. A mesma é propicia as temperaturas mediais anuais, acima da mínima requerida de $170 \mathrm{C}$, sendo esta região ponderada como das mais favoráveis para infestações de carrapatos (SANTANA, 2000).

Os carrapatos, atualmente têm grande relevância em relação à questão da Saúde Pública, pois algumas espécies destes vetores são responsáveis por transmitir várias enfermidades (SILVA e BRAGA, 2010).

A principal estratégia utilizada no controle deste ectoparasita, atualmente vem sendo o uso de acaricidas, a base de produtos químicos sintéticos. Entretanto, o controle tradicional por meio de carrapaticidas sintéticos gera problemas já conhecidos, como resistência, danos à natureza e à saúde humana, além de apresentar custos elevados ao criador, despertando a necessidade de desenvolvimento de novas alternativas para o controle deste ectoparasita (SPICER, 1993).

A resistência aos carrapaticidas comerciais surgiu como um problema em vários países, especialmente com relação aos carrapatos Rhipicephalus (Boophilus) microplus (Canestrini, 1887), Rhipicephalus sanguineus (Latreille, 
FERNANDES, T.F. e BRAGA, G.M.S. Obtenção de extratos liquênicos do Cerrado Maranhense para avaliação da atividade carrapaticida. PUBVET, Londrina, V. 8, N. 21, Ed. 270, Art. 1799, Novembro, 2014.

1806), Amblyomma cajennense (Fabricius, 1787) e Anocentor nitens (Neumann, 1897) (SILVA et al., 2005).

O uso incorreto do carrapaticida, como as subdoses, o preparo inadequado, a aplicação mal feita, faz com que os carrapatos não faleçam após contato com o produto. Cada vez que os carrapatos sobrevivem, a uma determinada aplicação de carrapaticida, eles transmitem as gerações posteriores, informações genéticas de como sobreviver aquele produto (FURLONG e PRATA, 2006).

Pela capacidade de desenvolvimento de resistência aos carrapaticidas, verifica-se redução gradativa na eficácia dos produtos. Tais fatores incentivaram a busca por outras formas de controle, mais incisivamente a partir da década de 90 . Dentre essas, o uso de fitoterápicos se destaca devido à grande variabilidade de espécies existentes, baixo custo, fácil disponibilidade na propriedade e, principalmente, pela baixa contaminação do ambiente e, em consequência, dos animais e do homem (HEIMERDINGER, 2005).

Hernández et al. (1987) relatam que, o uso de produtos naturais poderia minimizar o desequilíbrio ecológico e a contaminação ambiental, causada pelo uso intensivo de produtos químicos sintéticos. Com relação ao carrapato, diversas plantas têm sido utilizadas, nas pesquisas de programas de controle, tais como o Neem (Azadirachta indica) (MARTINEZ, 2002), o capimgordura (Melinis multiflora Beauv.) (PRATES et al., 1993) e algumas espécies do gênero Stylosanthes (CASTREJón et al., 2003), na tentativa de redução da utilização de produtos acaricidas sintéticos.

Como elemento essencial, de uma das alternativas de controle de artrópodes é que se está testando o líquen que é uma associação simbiótica mutualista entre um componente fúngico denominado de micobionte, e um componente fotossintetizante denominado fotobionte (RAVEN, 2007).

Até 1981, os liquens eram considerados como formando um grupo taxonômico, Lichenes, dentro do Reino Fungi. A partir de então, o Código Internacional de Nomenclatura Botânica (CINB), seguindo a prática já corrente 
FERNANDES, T.F. e BRAGA, G.M.S. Obtenção de extratos liquênicos do Cerrado Maranhense para avaliação da atividade carrapaticida. PUBVET, Londrina, V. 8, N. 21, Ed. 270, Art. 1799, Novembro, 2014.

entre os especialistas em liquens, aboliu Lichenes, como grupo taxonômico, que passou a ser encarado como um grupo biológico, com características fisiológicas e ecológicas próprias (MARCELLI, 1997).

Os liquens produzem metabólitos secundários denominados "substâncias liquênicas", antigamente chamadas de ácidos liquênicos. A maioria dessas substâncias é de natureza fenólica e são elas as responsáveis por grande parte das atividades biológicas atribuídas aos liquens (HALE, 1983).

\section{MATERIAL E MÉTODOS}

A área estudada foi a do Cerrado Maranhense, nas proximidades no município de Imperatriz, região Sudoeste do Estado do Maranhão, considerada Pré Amazônica, localizada no Nordeste brasileiro, a Oeste do meridiano de Greenwich, abaixo da linha do equador. Quanto ao aspecto físico, possui uma superfície de aproximadamente $1.368 \mathrm{Km}^{2}$ altitude de $95 \mathrm{~m}$, Latitude de $5^{\circ}$ $31^{`} 35^{\prime}$ 'Sul e Longitude de $47^{\circ} 29^{\prime} 30^{\prime}$ 'Oeste (MAPSAT, 2006).

O estudo foi realizado no período de agosto de 2011 a dezembro de 2012, sendo este o melhor tempo para coleta dos liquens para realização da pesquisa, sendo os mesmos do tipo folhoso ou foliáceo, que foram empregados para preparação dos extratos liquênicos, utilizados nos testes de avaliação em artrópodes parasitas.

Depois de coletados, os liquens foram acondicionados em sacos de papel opaco, e levados para o Laboratório de Biologia do Centro de Estudos Superiores de Imperatriz, na Universidade Estadual do Maranhão, LABIO. No mesmo foi realizada a catalogação dos espécimes e a identificação, mediante observação por meio de caracteres morfológicos e químicos do talo.

Após a identificação do material, foram produzidos os extratos por maceração do talo liquênico. $O$ isolamento dos compostos pelo sistema de esgotamento a frio, a partir dos solventes, álcool e acetona, para análise fotoquímica, foi de acordo com Santos et al., em 1997. 
FERNANDES, T.F. e BRAGA, G.M.S. Obtenção de extratos liquênicos do Cerrado Maranhense para avaliação da atividade carrapaticida. PUBVET, Londrina, V. 8, N. 21, Ed. 270, Art. 1799, Novembro, 2014.

Posteriormente ser realizada a vazão dos extratos, das amostras de liquens, foi efetivada a coleta e identificação de alguns organismos artrópodes parasitas de cão, bovinos e equinos, cito Rhipicephalus sanguineus, Rhipicephalus (Boophilus) microplus e Anocentor sp. coletadas no Centro Municipal de Controle de Zoonoses (CMCZ) e nas fazendas das proximidades do Município de Imperatriz, Maranhão. Nos mesmos foram realizados os testes in vitro, para avaliação da atividade carrapaticida dos extratos liquênicos.

A avaliação in vitro foi realizada de acordo com Santos et al. (2003), através dos organismos artrópodes conferidos e desempenhados, nos ensaios de biocontrole de teleóginas, de ovos e larvas.

Após a coleta, as fêmeas foram lavadas em água destilada e secas em papel toalha, colocadas em placa de Petri de forma homogênea, em grupo de dez, com a devida identificação, sendo pesadas e mantidas em temperatura ambiente por 24 horas (SOARES et al., 2001).

Depois deste período, as fêmeas foram selecionadas, sendo feita a eliminação das mortas ou as que tenham qualquer detrimento físico. Foram pesadas em balança analítica, as teleóginas homogêneas ingurgitadas, e colocadas em placa por cada espécie $\left(\mathrm{GC}_{1}\right)\left(\mathrm{GC}_{2}\right)\left(\mathrm{GC}_{3}\right)$ e grupo experimental por espécies correspondentes $\left(\mathrm{GE}_{1}\right)\left(\mathrm{GE}_{2}\right)\left(\mathrm{GE}_{3}\right)$ já citadas como Rhipicephalus sanguineus, Rhipicephalus (Boophilus) microplus e Anocentor sp. respectivamente.

No primeiro teste, as teleóginas do grupo controle foram submetidas a banhos de imersão, em $20 \mathrm{~mL}$ de álcool e cronometrado no tempo de 10 minutos. As espécies do grupo experimental foram imersas em $20 \mathrm{~mL}$ de solução de extrato liquênicos, preparados com álcool e cronometrado no mesmo período de tempo. Posteriormente, secas em papel toalha e depositadas em placa de Petri, identificadas e mantidas em estufa a $27^{\circ} \mathrm{C}$ de temperatura com umidade do ar acima de $75 \%$.

No segundo teste, consideradas do grupo controle foram imersas em $20 \mathrm{ml}$ de álcool e cronometrado no tempo de 20 minutos, a espécie do grupo experimental foi imerso em $20 \mathrm{ml}$ de solução de extrato liquênicos, preparado 
FERNANDES, T.F. e BRAGA, G.M.S. Obtenção de extratos liquênicos do Cerrado Maranhense para avaliação da atividade carrapaticida. PUBVET, Londrina, V. 8, N. 21, Ed. 270, Art. 1799, Novembro, 2014.

com álcool e cronometrado no mesmo período de tempo, sendo realizado o procedimento anterior.

No terceiro teste, consideradas do grupo controle foram submetidas a imersão de $20 \mathrm{ml}$ de álcool e cronometrado no tempo de 10 minutos. As espécies do grupo experimental $\left(\mathrm{GE}_{3}\right)$ foram imersas em $20 \mathrm{ml}$ de solução de extrato liquênicos, preparado com cinco $\mathrm{ml}$ de extrato e $15 \mathrm{ml}$ de álcool e cronometrado no mesmo período de tempo e em seguida realizado o mesmo processo.

Para a avaliação da eficácia do extrato foram utilizadas fórmulas de Drummond et al. (1971-1973), como teste "in vitro" das avaliações através de Biocarrapaticidograma.

A eclodibilidade das larvas foi avaliada por estimativa de porcentagem em relação àquelas que não eclodiram.

Com os resultados obtidos pelo peso das teleóginas, peso dos ovos e porcentagem de eclosão foi calculado a eficácia e ação carrapaticida de extratos liquênicos sobre os artrópodes Rhipicephalus (Boophilus) microplus (Canestrini, 1887), Rhipicephalus sanguineus (Latreille, 1806) e Anocentor sp. (Neumann, 1897).

\section{RESULTADOS E DISCUSSÃO}

Os liquens coletados foram classificados como Parmotrema sp. (MARCELLI, 1997) e no primeiro teste, após observação dos ovos foi notado que a espécie Riphicephalus sanguineus obteve cerca de $85 \%$ de eclosão dos ovos viáveis no grupo $\mathrm{GC}_{1}, 65 \%$ no $\mathrm{GE}_{1}$.

$\mathrm{Na}$ espécie Riphicephalus (Boophilus) microplus houve pequena eclosão dos ovos do grupo $\mathrm{GE}_{2}$, no grupo $\mathrm{GC}_{2}$ não houve oviposição. A espécie Anocentor sp. houve $90 \%$ de eclosão dos ovos viáveis no grupo $\mathrm{GC}_{3}$, e $90 \%$ no grupo $\mathrm{GE}_{3}$.

A relação do Índice de Eficiência Reprodutiva (IER) entre os Riphicephalus (Boophilus) microplus, Riphicephalus sanguineus e Anocentor sp. 
FERNANDES, T.F. e BRAGA, G.M.S. Obtenção de extratos liquênicos do Cerrado Maranhense para avaliação da atividade carrapaticida. PUBVET, Londrina, V. 8, N. 21, Ed. 270, Art. 1799, Novembro, 2014.

foi de valores entre $0,88 \%$ e $41 \%$ para Grupo Controle e com os valores $91 \%$, $68 \%$ e $61 \%$ para Grupo Experimental respectivamente.

O Índice de Eficácia do Produto (IEP), nas espécies em relação ficou entre os valores $76 \%$ e $48 \%$. Sendo assim, o Índice de Eficácia do Produto (IEP) foi melhor para o Riphicephalus sanguineus, como mostra a tabela 1.

Tabela 1 - Índices reprodutivos médios de fêmeas ingurgitadas de Riphicephalus (Boophilus) microplus, Riphicephalus sanguineus e Anocentor sp. avaliadas com extratos liquênicos extraídos de liquens da região do cerrado maranhense, 2012

\begin{tabular}{|c|c|c|c|}
\hline Espécie & IER GC & IER GE & IEP \\
\hline $\begin{array}{l}\text { Riphicephalus } \\
\text { (Boophilus) } \\
\text { microplus }\end{array}$ & $0,3 \%$ & $91 \%$ & $0,3 \%$ \\
\hline $\begin{array}{l}\text { Riphicephalus } \\
\text { sanguineus }\end{array}$ & $88 \%$ & $68 \%$ & $76 \%$ \\
\hline Anocentor sp. & $41 \%$ & $61 \%$ & $48 \%$ \\
\hline
\end{tabular}

IER: Índice de Eficiência Reprodutiva; IEP: Índice de Eficácia do Produto; GC: Grupo Controle; GE: Grupo Experimental.

No segundo teste, na espécie Riphicephalus (Boophilus) microp/us houve pequena eclosão do grupo controle $\left(\mathrm{GC}_{2}\right)$, porém não apresentou oviposição dos ovos. No grupo experimental $\left(\mathrm{GE}_{2}\right)$ as teleóginas amofinaram, sendo ocorrido em ambos os grupos, experimental $\left(G_{2}\right)$ e controle $\left(G_{2}\right)$, não ocorrendo oviposição dos ovos. O Índice de Eficácia do Produto (IEP) foi de 95\% em ambos os testes e após a observação dos ovos da espécie Anocentor sp. foi ressaltado que o $\mathrm{GC}_{2}$ obteve cerca de $90 \%$ de eclosão dos ovos viáveis, e $90 \%$ no $\mathrm{GE}_{2}$. O Índice de Eficiência Reprodutiva (IER) foi de $85 \%$ no $\mathrm{GC}_{2} \mathrm{e}$ $61 \%$ no $\left(\mathrm{GE}_{2}\right)$. O Índice de Eficácia do Produto (IEP) foi em torno de $28 \%$.

No terceiro teste, os ovos da espécie Riphicephalus (Boophilus) microplus apresentaram eclosão de $73 \%$ dos ovos viáveis no $\mathrm{GC}_{3}$ e $\circ \mathrm{GE}_{3}$ não houve eclosão. O Índice de Eficácia do Produto (IEP) foi de 95\%. Na espécie Riphicephalus sanguineus houve pequena eclosão do grupo controle $\left(\mathrm{GC}_{2}\right)$, porém não apresentou oviposição dos ovos. No grupo experimental $\left(\mathrm{GE}_{2}\right)$ as teleóginas amofinaram, sendo ocorrido em ambos os grupos, experimental $\left(G_{2}\right)$ e controle $\left(G_{2}\right)$, não ocorrendo oviposição dos ovos e o Índice de Eficácia do Produto (IEP) foi de 95\% 
FERNANDES, T.F. e BRAGA, G.M.S. Obtenção de extratos liquênicos do Cerrado Maranhense para avaliação da atividade carrapaticida. PUBVET, Londrina, V. 8, N. 21, Ed. 270, Art. 1799, Novembro, 2014.

Tabela 2 - Índices reprodutivos médios de fêmeas ingurgitadas de Riphicephalus (Boophilus) microplus e Anocentor sp. avaliadas com extratos liquênicos extraídos de liquens da região do cerrado maranhense, 2012

$\begin{array}{llll}\text { Espécie } & \text { IER Gc } & \text { IER Ge } & \text { IEP }\end{array}$

\begin{tabular}{cccc}
\hline $\begin{array}{c}\text { Riphicephalus } \\
\text { (Boophilus) } \\
\text { microplus }\end{array}$ & 0 & 0 & $95 \%$ \\
$\begin{array}{c}\text { Riphicephalus } \\
\text { sanguineus }\end{array}$ & 0 & 0 & $95 \%$ \\
Anocentor sp. & $85 \%$ & $61 \%$ & $28 \%$
\end{tabular}

IER: Índice de Eficiência Reprodutiva; IEP: Índice de Eficácia do Produto; GC: Grupo Controle; GE: Grupo Experimental.

Na espécie Anocentor sp. $\circ \mathrm{GC}_{3}$ apresentou eclosão de $62 \%$ dos ovos viáveis, e $56 \%$ no $\mathrm{GE}_{3}$. O Índice de Eficiência Reprodutiva (IER) foi de $61 \%$ no $\mathrm{GC}_{2}$ e $53 \%$ no $\left(\mathrm{GE}_{2}\right)$. O Índice de Eficácia do Produto (IEP) foi de $13 \%$, conforme a tabela 3.

Tabela 3 - Índices reprodutivos médios de fêmeas ingurgitadas de Riphicephalus (Boophilus) microplus e Anocentor sp. avaliadas com extratos liquênicos extraídos de liquens da região do cerrado maranhense, 2012

\begin{tabular}{|c|c|c|c|}
\hline Espécie & IER Gc & IER Ge & IEP \\
\hline $\begin{array}{l}\text { Riphicephalus } \\
\text { (Boophilus) } \\
\text { microplus }\end{array}$ & $62 \%$ & 0 & $95 \%$ \\
\hline $\begin{array}{l}\text { Riphicephalus } \\
\text { sanguineus }\end{array}$ & 0 & 0 & $95 \%$ \\
\hline Anocentor sp. & $61 \%$ & $53 \%$ & $13 \%$ \\
\hline
\end{tabular}

IER: Índice de Eficiência Reprodutiva; IEP: Índice de Eficácia do Produto; GC: Grupo Controle; GE: Grupo Experimental.

Devido às modificações do clima na região Sudoeste do Estado do Maranhão, que podem apresentar às vezes, altas temperaturas e baixa umidade, ou mesmo altas temperaturas vinculada à alta umidade houve período que, não foi possível realizar uma coleta significativa da espécie Riphicephalus sanguineus, no período estudado, devido aos fatores climáticos 
FERNANDES, T.F. e BRAGA, G.M.S. Obtenção de extratos liquênicos do Cerrado Maranhense para avaliação da atividade carrapaticida. PUBVET, Londrina, V. 8, N. 21, Ed. 270, Art. 1799, Novembro, 2014.

desempenharem acentuada influência no ciclo biológico dos carrapatos, especialmente na fase não parasitária.

Todavia, foi possível realizar um teste piloto apresentando este uma eficácia positiva em relação aos extratos liquênicos.

Silva et al. 2007, realizaram testes com diversas espécies de carrapatos usando extratos vegetais de Neem e Capim Santo, e de acordo com o referido pesquisador existe uma significativa eficiência destes extratos vegetais sobre os artrópodes.

Heimerdinger em 2005 realizou testes com extrato alcoólico de capim cidreira, onde a solução contendo $2,72 \%$ de capim cidreira apresentou baixa eficácia, porém proporcionou uma redução no número de teleóginas, corroborando com o presente estudo que, em alguns testes houve oviposição, porém não ocorreu eclosão dos ovos.

Micheletti et al. 2009 utilizaram as folhas de $A$. indica (Neem) e $C$. citratus (Capim-santo), sementes de $A$. muricata (Graviola) e flores de $S$. malaccensis (Jambo). Onde os extratos das sementes da graviola e da flor do jambo em concentração de $2 \%$ possuem significativo potencial de controle, causando a mortalidade das teleóginas nos primeiros dias após o tratamento, confirmando com este estudo, onde ocorreu morte das teleóginas antes de realizarem a ovipostura.

Deve-se observar que, a diminuição dos gastos na produção animal e a contaminação ambiental têm estado entre as principais justificativas, para a ampliação do uso alternativo do controle de artrópodes através de fitoterápicos.

\section{CONCLUSÃO}

Os extratos liquênicos da região Sudoeste do Cerrado Maranhense apresentam eficácia em alguns organismos artrópodes aracnídeos, quando o mesmo é dissolvido a partir dos solventes, álcool e acetona ou mesmo em estado puro. 
FERNANDES, T.F. e BRAGA, G.M.S. Obtenção de extratos liquênicos do Cerrado Maranhense para avaliação da atividade carrapaticida. PUBVET, Londrina, V. 8, N. 21, Ed. 270, Art. 1799, Novembro, 2014.

Estudos científicos que utilizam liquens no controle de ectoparasitas ainda são escassos, sendo este o primeiro estudo sobre a avaliação carrapaticida com extratos liquênicos, recomendando-se maiores investigações sobre o assunto.

\section{REFERÊNCIAS}

BARROS-BATTESTI, D. M., ARZUA, M., BECHARA, G. H., Carrapatos de Importância Médico-Veterinária da Região Neotropical: Um guia ilustrado para identificação de espécies. São Paulo, Vox/ICTTD-3-Butantan, p. 223. 2006.

CASTREJÓN, F. M., CRUZ-VASQUEZ, C., FERNÁNDEZRUVALCABA, M. Repellence of Boophilus microplus larvae in Stylosanthes humilis and Stylosanthes hamata plants. Parasitologia Latino americana. v.58, n.2-3, p.118-121, 2003.

DRUMMOND, R.O. et al. Laboratory testing of insecticides for control of the winter tick. Journal Economic Entomology. v. 64, p. 686-688. 1971.

DRUMMOND, R.O. et al. Boophilus annulatus and Boophilus microplus: Laboratory Tests of inseticides. Journal Economic Entomology., v. 66, p. 130-133. 1973.

FURLONG, J.; PRATA, M. Controle estratégico do carrapato dos bovinos de leite. Juiz de Fora: EMBRAPA-CNPGL. P.2, (Circular Técnica, 38). 2006.

HALE Jr., M. E. The Biology of Lichens. 3 rd ed. London: Edward Arnold, p. 90. 1983.

HEIMERDinger, A. Extrato Alcoólico de Capim-Cidreira (Cymbopogon citratus) no Controle do Carrapato (Boophilus microplus) de Bovinos Leiteiros. Santa Maria, 2005. 78 f. Dissertação (Mestrado em Zootecnia) - Centro de Ciências Rurais, Universidade Federal de Santa Maria (UFSM - RS), Santa Maria. 2005.

HERNÁNDEZ, L. E.; PARRA, D. G.; MARIN, A. C. Ación repelente y acaricida del Melinis minutiflora sobre el Boophilus microplus. Revista Colombiana de Ciencias Químico Farmacéuticas, v.16, p.17-21, 1987.

MAPSAT - Tecnologia em Geoprocessamento. Disponível em: <www.mapsat.com.br.> Acesso em 01/12/2012.

MARCELLI, M. P. Estudo da diversidade de fungos liquênicos do Estado de São Paulo. Instituto de Botânica Secção de Micologia e Liquenologia, São Paulo. 1997.

MARTINEZ, S. S. O emprego do Nim. In: MARTINEZ, S. S. Anais... O Nim - Azadirachta indica: natureza, usos múltiplos, produção. Londrina: IAPAR, p.69-80. 2002.

MICHELETTI, S.M.F.B. et al. Extratos de plantas no controle de Rhipicephalus (Boophilus) microplus (Canestrini, 1887) (Acari: Ixodidae) em laboratório. Revista Brasileira de Parasitologia Veterinária, Jaboticabal, v. 18, n. 4, p. 44-48, out.-dez. 2009.

PRATES, H. T., OliVeirA, A. B., LEITE, R. C., CRAVEIRO, A. A. Atividade carrapaticida e composição química do óleo essencial do capim-gordura. Pesquisa Agropecuária Brasileira, Brasília, v.28, n.5, p.621-625, Maio, 1993. 
RAVEN, P. H.; EVERT, R. F. et al. Relações Simbióticas em Fungos. Biologia Vegetal. Rio de Janeiro, RJ. Guanabara Koogan, p. 302-307, 2007.

SANTANA, V.L.A. Situação do controle químico do Boophilus microplus (CANESTRINI, 1887) das sub-regiões da Zona da Mata e Agreste do Estado de Pernambuco com base em testes "ïn vitro" da eficácia de carrapaticidas em fêmeas ingurgitadas. 2000. Dissertação de Mestrado em Ciência Veterinária. UFRPE, Recife - PE. 2000.

SANTOS et al. Efeito da sazonalidade na produção de metabólitos com ação antitumoral em Cladonia verticillaris (Líquen). Revista da Universidade do Amazonas, Série Ciências Biológicas, Manaus. v. 2. n.2. 1997.

SANTOS, R.A. et al. Avaliação in vitro de carrapaticidas em amostra de fêmeas ingurgitadas de Boophilus microplus do município de Água Branca-AL. In: Congresso Pernambuco de Medicina Veterinária, 5, p. 352-353, 2003.

SILVA, W. W. et al. Resistência de fêmeas ingurgitadas de Boophilus microplus e Riphicephalus sanguineus (Acari: Ixodidae) a carrapaticidas no semi-árido paraibano: efeito da cipermetrina e do amitraz. Athayde/Agropecária Cietífica no Semi-árido, v. 01. p. 59-62, 2005.

SILVA, W.W. et al. Efeitos do Neem (Azadirachta indica A. juss) e do Capim Santo (Cymbopogon citratus (DC) Stapf) sobre os parâmetros reprodutivos de fêmeas ingurgitadas de Boophilus microplus e Rhipicephalus sanguineaus (Acari: Ixodidae) no semiárido paraibano. Revista Brasileira de Plantas Medicinais, Botucatu, v. 9, n.3, p.1-5, 2007.

SILVA, O. A.; BRAGA, G. M. S. O papel do Rhipicephalus sanguineus na transmissão da Leishmaniose Visceral Canina: aspectos epidemiológicos. PUBVET, v. 4, n. 25, 2010.

SOARES, V. E. et al. Análise in vitro da ação de carrapaticidas em cepas de Boophilus microplus (Canestrine 19870) colhidas de bovinos leiteiros da região nordeste do estado de São Paulo, Brasil Semina: Ciências Agrárias, Londrina, v. 22, n.1, p. 85-90. 2001.

SOUTELLO, R. V. et al. Seleção de bovinos de corte resistentes ao carrapato. Ciências Agrárias da Saúde. FEA, Andradina, v. 2, n. 2. p 57-60. 2002.

SPICER, P.E.; KEREU, R.K. Organochlorine inseticide residues in human breast milk: a survey of lactating mothers from a remote area in Papua New Guinea. Bulletim of Environmental Contamination and Toxicology. v. 50, p.540-546, 1993. 